\title{
DESDE LA PLANEACIÓN HASTA LA FABRICACIÓN DE PRÓTESIS DE CADERA PERSONALIZADAS POR MANUFACTURA ADITIVA DE METALES
}

\author{
Jorge Corona-Castuera ${ }^{1 *}$, Daniela Rodríguez-Delgado ${ }^{2}$, John Henao $^{3}$, Carlos A. \\ Poblano-Salas 4
}

1: Investigador, CIATEQ A.C., Av. Manantiales 23-A, Parque Industrial Bernardo Quintana, El Marqués, Querétaro, México.

2: Ingeniero biomédico, CIATEQ A.C., Av. Manantiales 23-A, Parque Industrial Bernardo Quintana, El Marqués, Querétaro, México

3: Catedrático, CONACYT-CIATEQ, Av. Manantiales 23-A, Parque Industrial Bernardo Quintana, El Marqués, Querétaro, México.

4: Investigador, CIATEQ A.C., Av. Manantiales 23-A, Parque Industrial Bernardo Quintana, El Marqués, Querétaro, México

*E-Mail: jcorona@ciateq.mx

\begin{tabular}{ll}
\hline PALABRAS CLAVE & RESUMEN \\
\hline Manufactura aditiva & La artroplastia total de cadera es uno de los procedimientos quirúrgicos \\
Prótesis de cadera & más comunes a nivel mundial en pacientes adultos; este procedimiento \\
Personalización & implica el reemplazo de la cadera por un implante cuyo objetivo es \\
Diseño & restablecer la movilidad de la extremidad afectada. La planeación de la \\
& intervención quirúrgica, así como el diseño de la prótesis son \\
& fundamentales para el éxito del implante. El presente trabajo presenta \\
& una revisión de la planeación, diseño y selección de una prótesis de \\
& cadera, así como de la consecución de una prótesis personalizada hoy \\
& en día posible gracias a las técnicas de manufactura aditiva. \\
\hline
\end{tabular}

\section{FROM PLANNING TO THE MANUFACTURING OF HIP PROSTHESIS BY ADDITIVE MANUFACTURING}

\begin{tabular}{ll}
\hline KEYWORDS & ABSTRACT \\
\hline Additive manufacturing & Total hip arthroplasty is one of the most common surgical procedures \\
Hip prosthesis & worldwide in adult patients; This procedure involves the replacement \\
Customization & of the hip with an implant whose objective is to restore mobility to \\
Design & the affected limb. The planning of the surgical intervention, as well \\
& as the design of the prosthesis are fundamental for the success of the \\
& implant. This paper presents a review of the planning, design and \\
& selection of a hip prosthesis, as well as the achievement of a \\
& personalized prosthesis that is currently possible thanks to additive \\
& manufacturing techniques. \\
\hline
\end{tabular}




\section{INTRODUCCIÓN}

Diseñar e imprimir implantes personalizados se ha convertido en una realidad durante los últimos 20 años. Muchas veces los dispositivos y servicios médicos son muy costosos y no están disponibles para todos los pacientes. Cualquier instrumento o producto médico pasa por un elaborado proceso de aprobaciones y ensayos clínicos, incluso los pacientes que pueden pagar el implante $\mathrm{u}$ órgano a veces no pueden encontrar el que sea adecuado para ellos. Además, el proceso de fabricación habitual de los implantes puede tardar días o semanas.

Las tecnologías de manufactura aditiva relativamente nuevas y el rápido desarrollo de software para la reconstrucción digital en 3D de modelos anatómicos a partir de imágenes médicas, han abierto oportunidades para el diseño y la fabricación de implantes específicos para pacientes. Con base en la información de una tomografía computarizada (CT) o resonancia magnética (MRI) de un paciente, se pueden producir prótesis de cualquier complejidad, tamaño y forma [1].

La personalización al adaptar un producto para satisfacer una necesidad específica se ha convertido en un área de gran interés en la industria médica. Hoy en día, muchas aplicaciones biomédicas se basan en productos personalizados, como prótesis e implantes que reemplazan miembros o huesos perdidos, o que reconstruyen una función articular específica para la situación del paciente. Otros ejemplos son los aumentos óseos o reemplazos óseos parciales, los componentes de la diáfisis de las extremidades o las réplicas anatómicas completas [2].

Debido a la cadena de proceso digital, es factible aplicar diseños personalizados y específicos del paciente para tratar de manera óptima la afección médica; una propuesta de la cadena de proceso para prótesis e implantes puede incluir entre otros (ver Figura 1) los requisitos del producto o necesidades del paciente, los cuales están delimitados por sus respectivos casos clínicos.

El proceso de diseño y producción de implantes, basado en la ingeniería inversa a partir de las imágenes o escaneos (CT, MRI), es extremadamente regulado por las autoridades sanitarias y normas y es aquí donde la manufactura aditiva cobra importancia, pues para dispositivos médicos la producción deberá apuntar a una producción especificada y validada, permitiendo una trazabilidad completa y asegurando la calidad del producto. La etapa de post procesamiento depende del diseño, el material y el proceso de fabricación empleado, algunos materiales metálicos requerirán tratamientos térmicos, y finalmente los productos deben esterilizarse $[2,3]$

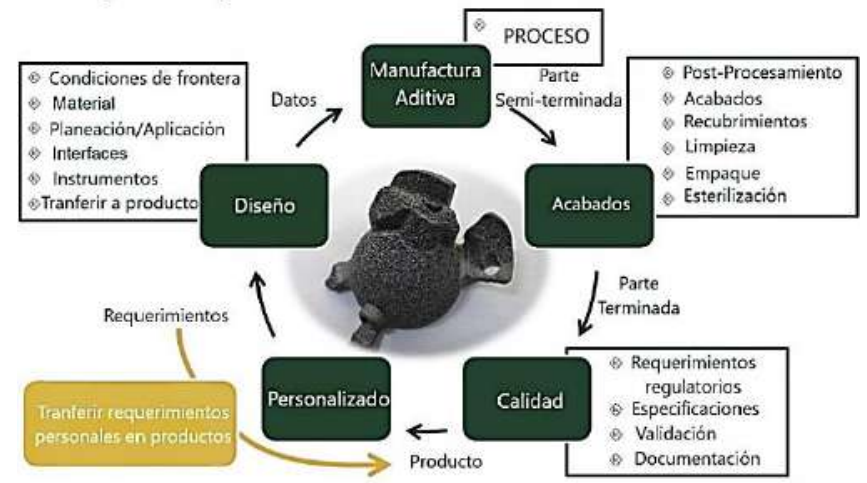

Figura 1. Cadena de proceso para prótesis e implantes [2].

Las tecnologías de manufactura aditiva tienen el potencial de causar una revolución en la cirugía ortopédica. La medicina individualizada parece cada vez más cerca de hacerse realidad, sin embargo, la consolidación en la práctica clínica y académica diaria depende de si los avances futuros podrán superar las desventajas aún presentes, tales como los costos de las 
impresoras 3D, la velocidad y precisión de impresión, así como la calidad.

Los objetos fabricados con tecnología de impresión 3D siguen siendo inferiores en calidad a los tradicionales, por lo que deben mejorarse las propiedades mecánicas de los objetos impresos; por otro lado, la seguridad de la tecnología de manufactura aditiva, así como, las directrices y regulaciones que controlan las aplicaciones son muy necesarias en el futuro próximo [4].

\section{PLANEACIÓN}

\section{PREOPERATORIA EN ARTOPLASTÍA DE CADERA}

La planeación preoperatoria ha sido parte fundamental en procedimientos totales $\mathrm{o}$ parciales de cadera. La planeación permite establecer el tamaño correcto del implante, el tipo de implante, disminuir riesgos de fractura peri-protésica, facilita la optimización en la alineación de la prótesis, ayuda a evitar o disminuir diferencias entre la longitud de ambas piernas y a reducir costos asociados con posibles riesgos con respecto a una cirugía no planeada [5].

Es importante mencionar que el objetivo de una cirugía de reemplazo de cadera es la reducción de dolor y restauración de la movilidad con el fin de permitir al paciente el retorno a sus actividades cotidianas. Por consiguiente, desde el ámbito médico es crucial realizar un análisis adecuado del padecimiento, así como de la anatomía del paciente para sugerir el implante con las mejores prestaciones posibles. Cuando factores como la orientación o el tamaño del implante no son los adecuados, suelen resultar complicaciones que se manifiestan con dolor, desgaste superficial de la prótesis, dificultad para caminar, y aumento en la probabilidad de fractura del hueso periprotésico [6].

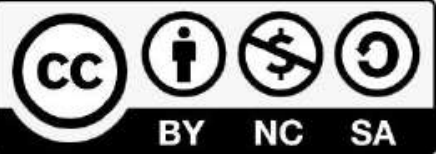

La planeación preoperativa para artroplastia de cadera tradicionalmente se ha realizado mediante el uso de "plantillas" superpuestas a imágenes en 2D [7]; este método consiste en la adquisición de radiografías digitales en la parte anteroposterior de la pelvis. Las imágenes adquiridas son empleadas para sobreponer una plantilla con un diseño predeterminado comercial de prótesis con tamaños estándares. El primer paso de esta metodología consiste en reconocer la magnificación de la radiografía con el fin de mantener las proporciones en las distancias y tamaños anatómicos. Normalmente este reconocimiento se realiza a partir de un implante preexistente de tamaño conocido o colocando un marcador radiográfico de tamaño conocido a un costado del paciente durante la toma de imágenes; a partir de estas imágenes, se determina la orientación del eje de la pelvis empleando la radiografía anteroposterior. Este eje es tomado como referencia para la comparación entre las piernas derecha e izquierda del paciente.

Para dibujar este eje, la orientación de la pelvis debe ser previamente identificada utilizando marcadores, los cuales tradicionalmente son la lágrima acetabular, la tuberosidad isquiática, el foramen obturador o la muesca ciática mayor. Se debe mencionar que el marcador de referencia más común es la lágrima acetabular.

Cuando estos marcadores están conectados por una línea, se forma un eje que sirve de referencia para la orientación de la pelvis. Este eje marca el ángulo de inclinación del componente acetabular; como resultado se puede conocer la posición adecuada para el implante en relación con la pelvis. Adicionalmente, se puede establecer si se presenta una discrepancia en la longitud de las extremidades. Esto se determina midiendo un punto sobre el fémur perpendicular al eje de la pelvis y comparando las medidas entre el lado izquierdo y derecho. Se pueden 
utilizar muchos puntos de referencia, incluido el trocánter mayor y menor. Con mayor frecuencia, se selecciona la parte superior del trocánter menor, ya que se trata de un punto de referencia que se identifica fácilmente radiográficamente. En este punto, se puede conocer si las longitudes de las extremidades son clínicamente iguales y si es pertinente o no la corrección en la longitud de las extremidades al colocar el implante [8].

Adicionalmente, se debe determinar el centro de rotación de la articulación de la cadera colocando la plantilla del componente acetabular del implante digitalizado, en el centro dentro de los límites del acetábulo en un ángulo de aproximadamente $45^{\circ}$ con respecto al eje pélvico. Los puntos de referencia óseos incluyen el labio superior lateral e inferolateral del acetábulo con el borde medial de la prótesis colocado dentro de las paredes internas y externas de la pelvis $[8,9]$.

Por otra parte, también este procedimiento permite determinar el tamaño del componente femoral. Esto se logra midiendo el ancho del canal endoóseo distalmente dentro de la diáfisis femoral y el ancho del canal endoóseo proximal dentro de la metáfisis; en consecuencia, se selecciona un tamaño apropiado de prótesis que se ajuste mejor a los límites del fémur empleando la plantilla predefinida del implante [8].

Es importante mencionar que el tamaño del componente femoral apropiado se selecciona superponiendo la imagen de la plantilla dentro del fémur en una posición que reproduzca la longitud de la extremidad existente (si no existe una discrepancia en la longitud de la extremidad) o para corregir cualquier discrepancia en la longitud de la extremidad; además, es crucial que en este procedimiento se asegure que el implante seleccionado también reproduzca adecuadamente el desplazamiento femoral de la cadera. Finalmente, se debe calcular la longitud de la resección (corte quirúrgico) del cuello femoral, midiendo desde la parte superior del trocánter menor al trocánter mayor. Esta longitud sirve de referencia para colocar el componente femoral en posición de acuerdo con la copa acetabular seleccionada $[8,10]$.

De manera general, la metodología preoperatoria basada en "plantillas" permite conocer el tamaño apropiado del implante de cadera (cabeza femoral, vástago, longitud de cuello, tamaño de la copa acetabular, corrección de longitud de extremidades en caso de requerirse) y de la resección ósea en una cirugía de cadera con el fin de brindar las mejores prestaciones posibles para el paciente.

La metodología antes descrita ha sido ampliamente usada en hospitales alrededor del mundo durante décadas y con el desarrollo de nuevas tecnologías esta metodología ha pasado de ser meramente manual a digitalizarse. Hoy en día se emplean softwares que permiten de forma digital analizar la radiografía y superponer una "plantilla" precargada en una base de datos de diseño de prótesis con tamaño predefinido disponible en el mercado $[11,12]$. Incluso este tipo de softwares incluyen algoritmos que reducen el número de mediciones en el software pero que entregan las distancias y mediciones que se describieron previamente para la selección del implante. Con la digitalización de las imágenes radiográficas es entonces tarea del médico seleccionar a partir de la biblioteca digital de plantillas con los diseños de la prótesis aquella más acorde a las necesidades del paciente.

En la última década, se ha identificado que, al utilizar implantes no cementados, se han presentados hasta un $62 \%$ de casos con una discrepancia hasta de $1 \mathrm{~cm}$ en las extremidades en pacientes tratados con una 
planeación preoperatoria basada en plantillas 2D [13], lo cual provoca inconformidades y dificultades en la recuperación de los pacientes. En este sentido, en años recientes se ha buscado mejorar la planificación preoperatoria pasando de la digitalización 2D a la planificación computarizada 3D con el fin de lograr una mayor precisión en la selección del implante con respecto a las plantillas 2D convencionales $[14,15]$.

Se han desarrollado softwares comerciales (p. ej, hipEOS®) los cuales realizan la reconstrucción $3 \mathrm{D}$ de la zona afectada a partir de imágenes 2D adquiridas por radiografía. Este tipo de softwares se basan en la adquisición simultánea de imágenes radiográficas ortogonales; las imágenes obtenidas se modelan posteriormente en 3D empleando algoritmos integrados en el software. Este modelo 3D de la cadera del paciente se contrasta con plantillas predefinidas de implantes comerciales en 3D para realizar la selección de los componentes más adecuados para el paciente [14].

De forma alternativa, también se ha empleado la tomografía computarizada $3 \mathrm{D}$ para la planificación preoperatoria [16, 17]. Esta técnica también consiste en la aplicación de rayos $\mathrm{X}$ en la zona de estudio del paciente. En dicha técnica la diferencia radica en que se gira rápidamente la aplicación de la radiación alrededor del cuerpo, produciendo señales que son procesadas por la computadora de la máquina para generar imágenes transversales de la zona irradiada del cuerpo [18]. Estos cortes pueden ser tratados a través de softwares especializados para obtener digitalmente una reconstrucción 3D de la cadera del paciente.

Independientemente de la técnica de reconstrucción $3 \mathrm{D}$, se ha documentado que la reconstrucción ósea 3D resulta en una mejor precisión que la planificación por plantillas en $2 \mathrm{D}$, en particular, con una mejor precisión en la restauración de longitudes de las extremidades y del offset femoral. En las plantillas 2D, la tasa de precisión para los componentes no cementados varía entre el $20 \%$ y el $70 \%$ [10, 19-21], sin embargo, la planificación 3D, [14] presenta una precisión por encima del $90 \%$.

Uno de los principales intereses de la planificación preoperatoria $3 \mathrm{D}$ es la anticipación de las dificultades quirúrgicas, de hecho, la planificación 3D permite el análisis de anormalidades, especialmente la torsión femoral y una mejor evaluación del desplazamiento femoral [22]. En años recientes, la reconstrucción ósea en 3D se ha empleado en la planificación preoperatoria no solo para seleccionar el tipo de implante más adecuado para los pacientes con padecimientos en la cadera sino también para planificar la intervención quirúrgica [23]. Es importante mencionar que algunas compañías ofrecen equipo de manufactura aditiva especializado en la reconstrucción ósea a partir de imágenes en 3D del paciente para producir prototipos base polimérica. Los prototipos del hueso en polímero son usualmente empleados como modelos reales del hueso del paciente para ensayos pre quirúrgicos en donde los cirujanos pueden ensayar previamente la colocación del implante. Esta nueva tendencia ha contribuido no solo a mejorar la selección de los implantes sino también a disminuir los tiempos de las cirugías [23, 24].

Es importante mencionar que la planificación preoperatoria, aunque juega un rol importante en la artroplastia de cadera por los varios motivos médicos ya antes expuestos, también revela una de las problemáticas más importantes en las cirugías de cadera, esto es las dimensiones predeterminadas de los implantes. Esto implica que el uso de "plantillas" con diseños predeterminados llevan a la selección de tamaños de prótesis que más se ajusten al paciente y que resultan 
en procedimientos que requieren resecciones óseas significativas para ajustar el tamaño predeterminado de la prótesis a la cadera y hacer coincidir las dimensiones de las extremidades. En este sentido, la experiencia con la planeación preoperatoria ha llevado en años recientes a proponer la manufactura aditiva en 3D, no solo para la generación de prototipos de la cadera para ensayo quirúrgico, sino también para el diseño de prótesis con dimensiones ajustadas a la anatomía del paciente; es decir, para el diseño de las denominadas "prótesis personalizadas".

\section{PERSONALIZACIÓN PRÓTESIS DE CADERA}

DE

La personalización de una prótesis se fundamenta en el estudio de la estructura anatómica del paciente, en particular, de la región en la que se busca recuperar la movilidad y/o mejorar la condición física. Como resultado del estudio anatómico se busca un diseño de prótesis que se ajuste fielmente a la anatomía del paciente. De esta manera, una de las etapas más importantes en la personalización de prótesis es el diagnóstico clínico y reconstrucción anatómica del hueso o extremidad mediante técnicas como el CT-Scan. El CT-scan permite obtener una serie de imágenes en formato DICOM (Digital Imaging and Communication in Medicine), que contiene información en 2D de la región del cuerpo escaneada. Por medio del uso de software especializados en el tratamiento de imágenes DICOM, es posible extraer el conjunto de imágenes $2 \mathrm{D}$ en un modelo tridimensional que permita emular la estructura anatómica del paciente, y que, a su vez, sirva como modelo base para el diseño de la prótesis a la medida del paciente.

Para ejemplificar la ruta de personalización de una prótesis, en la Figura 2 se muestra cada uno de los pasos empleados para el desarrollo de una prótesis de cadera. Es importante mencionar que dependiendo de las características que busque el diseñador en la prótesis, alguno o varios de los pasos mostrados en la Figura 2 pueden ser trabajados a mayor profundidad y/o detalle. Por ejemplo, en el cálculo de la densidad ósea del paciente, se pueden extraer tantos datos como sea posible, con el fin de obtener un valor de densidad ósea confiable. Así mismo, en el diseño de la estructura con densidad variable se puede realizar una elección basada en la estructura ósea y resistencia mecánica tratando de emular las propiedades mecánicas del hueso femoral.

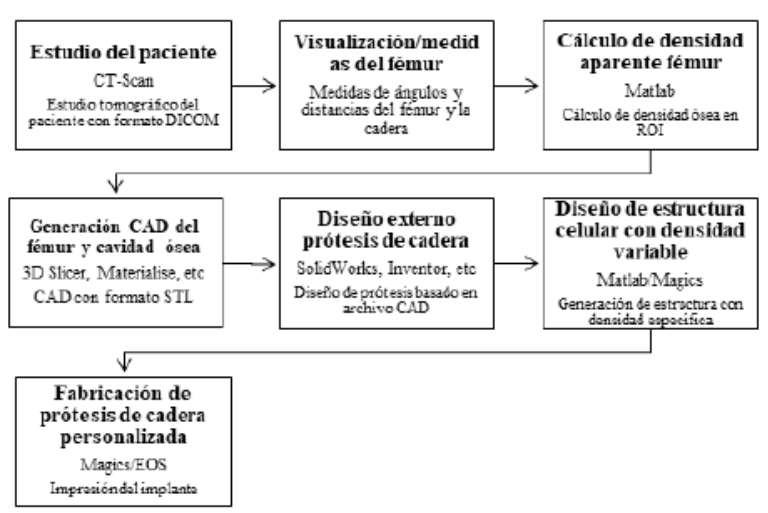

Figura 2. Proceso para diseño y fabricación de un implante de cadera personalizado.

El primer paso para el diseño de una prótesis personalizada, de acuerdo con la Figura 2, es el análisis médico del paciente para realizar un diagnóstico que permita decidir qué tipo de estudio será más adecuado para la recolección de datos anatómicos conforme a la afección del paciente. La tomografía computarizada es considerada el mejor método cuando se desea extraer modelos tridimensionales de tejido óseo.

Los pasos principales para el procesamiento de imágenes son los siguientes [25]:

1. Realzamiento.

2. Segmentación.

3. Cuantificación. 
4. Registro.

5. Visualización.

La calidad de las imágenes dependerá de diversos factores empleados al momento de realizar el estudio, como la cantidad de energía empleada, el espesor de corte asignado, entre otros, ayudando a tener una mejor resolución de las imágenes, por lo que es recomendado tener una buena comunicación con el especialista que realizará el estudio.

Una vez realizado el estudio es necesario cargar las imágenes para su visualización; existen diversos softwares de uso libre, así como privados que permiten visualizar y tratar imágenes DICOM. Un ejemplo de este tipo de software es aquel de uso libre llamado MiTK, el cual permite visualizar el conjunto de imágenes 2D de forma tridimensional para realizar su procesamiento; también permite realizar medidas de las imágenes en cualquier plano anatómico. A pesar de que el diseño se basa en los modelos tridimensionales del hueso, es importante tomar en cuenta los ángulos y distancias más importantes para el diseño de prótesis, con la finalidad de asegurar el cumplimiento de las recomendaciones marcadas por las normas internacionales para el diseño de prótesis de cadera; así como para asegurar que el diseño cumpla con las dimensiones registradas para el hueso de cada paciente.

Dependiendo de las características buscadas mecánicamente en la prótesis, se puede emplear un método para conocer la distribución de densidades del hueso del paciente, misma que puede replicarse de diversas formas, una de ellas es mediante la inclusión de estructuras celulares que permitan emular la densidad de cierta región del hueso, y el comportamiento mecánico del mismo [25, 26, 27].
Diversos autores han estudiado métodos para calcular la densidad aparente del tejido óseo mediante el cálculo de las unidades Hounsfield inmersas en las imágenes tomográficas [28, 29]. Las unidades Hounsfield determinan el nivel de atenuación que presenta un material ante el paso del haz de rayos $\mathrm{X}$, obteniendo para el tejido óseo niveles de entre 270 a 3000 unidades. Una parte importante de este tipo de análisis es el cálculo de la densidad radiológica $\left(\rho_{Q C T}\right)$, misma que se determina mediante el valor promedio de unidades Hounsfield para cada vóxel dentro del volumen tomográfico. Esto hace posible obtener una aproximación de la densidad aparente para tejido trabecular y cortical. De esta manera se puede establecer una densidad para las diferentes regiones anatómicas del fémur en su parte proximal, es decir, es posible calcular la densidad de la cabeza femoral, del cuello femoral, trocánter mayor, trocánter menor, diáfisis femoral, etc.

El siguiente paso para diseñar una prótesis de cadera personalizada, según la Figura 2, es la reproducción $3 \mathrm{D}$ del fémur mediante la reconstrucción del conjunto de imágenes DICOM del paciente. Es posible encontrar en el mercado diferentes softwares, tanto de uso libre como de pago, que permiten tratar imágenes médicas; un ejemplo de este tipo de softwares es el 3D Slicer, el cual es de uso libre y fue creado desde hace 2 décadas con el apoyo de "the National Institute of Health" y comunidades de desarrolladores alrededor del mundo. En este paso, con el fin de personalizar la prótesis, y con ayuda del software, se busca reconstruir el hueso del paciente con el propósito de obtener una base anatómica para el diseño de la prótesis.

En la Figura 3 se presenta un diagrama de flujo típico relacionado con la reconstrucción del fémur de un paciente empleando el software 3D Slicer. 
Este tipo de procesamiento de imágenes se basa en los siguientes pasos:

1. Realzamiento; se definen los límites entre diferentes tejidos representados en cada imagen DICOM.

2. Segmentación; se aplican transformaciones para lograr el agrupamiento de pixeles en cada imagen, por lo que cada grupo reúne los pixeles representados por el mismo material (hueso, músculo, grasa, vasos). La mayoría de los softwares empleados permiten seleccionar un intervalo de umbral para poder reunir los pixeles con valores similares de $\mathrm{HU}$, mismos que corresponden a un mismo tejido. También es posible aplicar la remoción de ruido inmerso en la imagen, este tipo de ruido se puede deber a diferentes factores, como (la generación de artefactos producidos, ya sea por el movimiento del paciente al realizar el estudio, penumbras geométricas por mala alineación de los rayos $\mathrm{X}$, por alteración de los detectores, entre otros; o debido a la aparición de HU similares de tejidos circundantes.

3. Visualización; al terminar el modelo de reconstrucción tridimensional se puede visualizar para asegurar que cumpla con la geometría deseada y corroborar la superficie. Por último, es posible exportar el modelo en formato STL, para su post-procesamiento.

La reconstrucción de archivos CAD del hueso se puede realizar de una o más estructuras anatómicas, dependiendo de las necesidades del diseñador, para el caso de la cadera, es posible generar hasta 3 modelos, el primero reconstruye la geometría externa del hueso femoral, el segundo la porción de la cadera que conforma el acetábulo, y por último, se puede reconstruir la cavidad femoral del paciente. Con estas tres estructuras se puede garantizar una personalización completa del implante de cadera, pues los modelos reconstruidos corresponden a la anatomía real del paciente.

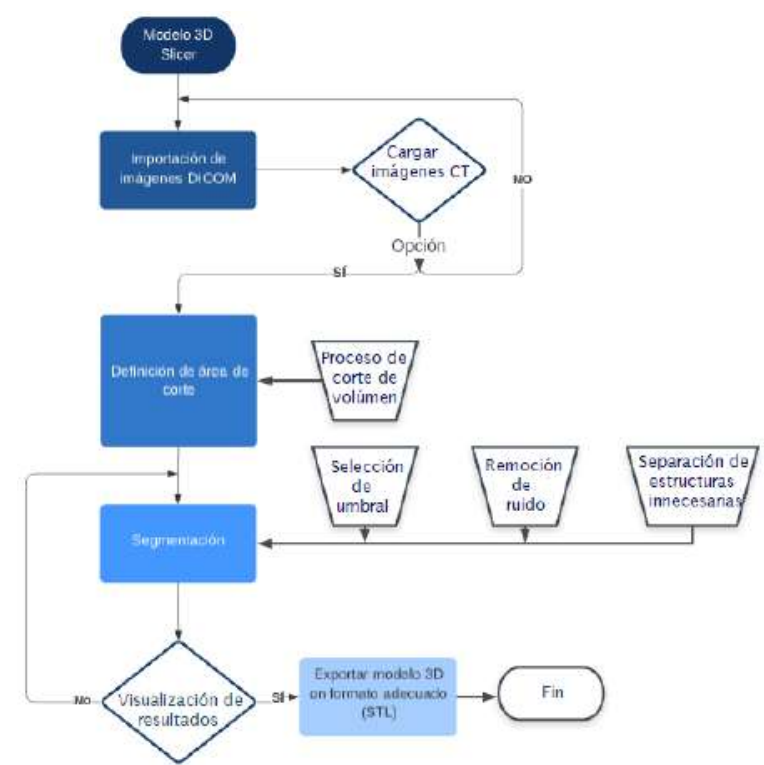

Figura 3. Diagrama de flujo para generar un archivo CAD mediante el tratamiento de imágenes médicas, empleando 3D Slicer.

Una vez generados los modelos 3D del hueso que servirán como base para el diseño del implante, se emplea un software CAD para diseño y modelado de la prótesis; existen diversas opciones de este tipo de softwares, como SolidWorks, CATIA, Inventor, entre otros. Este tipo de herramientas servirá para generar el diseño ajustado del implante dentro cde la cavidad ósea, permitiendo ajustar la geometría externa de la prótesis, y establecer medidas y ángulos específicos.

A continuación, una vez que se ha conseguido el diseño de la geometría externa del implante, se busca personalizar también su volumen interior con el propósito de obtener características mecánicas similares a las registradas por el tejido óseo. Este propósito se puede lograr empleando estructuras celulares (porosas) con propiedades específicas que emulen la densidad de las distintas regiones del hueso femoral. El diseño de la estructura celular puede realizarse mediante software de cómputo 
numérico como Matlab. Por ejemplo, la estructura denominada como "giróide simple triplemente periódico", es decir, que su geometría se replica de igual forma en los tres ejes, es una de las candidatas para este tipo de aplicaciones.

El uso de estructuras celulares tiene entonces como objetivo el control la densidad de la prótesis y busca reproducir las variaciones de densidad que el hueso natural presenta; es decir, se puede generar una estructura con densidad variable, mostrando un área con una densidad específica $\rho_{1}$, la siguiente con una densidad $\rho_{2} \quad y$ así sucesivamente, dependiendo de los valores de densidad calculados para las diferentes regiones del fémur. Una vez generada la estructura con densidad variable se inserta dentro del diseño de la prótesis y se exporta en el archivo final en formato STL.

Por último, con ayuda de un software de preparación y edición de archivos STL, como Magics de Materialise, se realiza un postprocesamiento que permite reparar errores en la geometría del diseño, el traslape de caras, agujeros en la estructura, entre otros, así como su preparación para la impresión generando los soportes adecuados para la fabricación del modelo.

Finalmente, empleando una herramienta de slicing, como RP-Tools de EOS, se realiza un análisis capa por capa del archivo corregido. A continuación, se exporta el archivo STL al software del equipo de manufactura aditiva para preparar la impresión del modelo, ajustando los parámetros de procesamiento, la posición y número de piezas sobre la placa de impresión. Finalmente, el archivo se envía a la impresora para comenzar su manufactura.

\section{MANUFACTURA DE PRÓTESIS PERSONALIZADAS POR ADITIVA}

La tecnología de diseño 3D implica la impresión de un solo material o una combinación de materiales capa por capa, regulando la forma de cada capa individual, lo que eventualmente resulta en una estructura 3D compleja con restricciones limitadas en su disposición espacial. Este tipo de tecnología ha mostrado una amplia variedad de aplicaciones en la industria médica, dando la posibilidad de imprimir estos modelos tridimensionales empleando diferentes tipos de materiales, según la necesidad y características que deba poseer el modelo y la técnica de fabricación empleada.

La tecnología de impresión 3D ha introducido sobre todo una gran cantidad de innovaciones en el ámbito musculoesquelético y enfermedades de la columna vertebral. La cirugía craneal y maxilofacial registran un $12.72 \%$ y $24.12 \%$ respectivamente, de las publicaciones relacionadas a aplicaciones quirúrgicas de la impresión 3D; mientras que ortopedia y cirugía vertebral muestran un $48.16 \%$ y $7.46 \%$ respectivamente [25].

En el año 2009, se formó un nuevo comité dedicado a la especificación de estándares para la manufactura aditiva (AM) llamado American Society for Testing and Materials (ASTM). Dicho comité categorizó todas las tecnologías de impresión 3D dentro de 7 grupos, mismos que se describen brevemente en la Tabla 1 [25]. 
Tabla 1. Categorización de tecnologías de impresión 3D según ASTM [25].

\begin{tabular}{|c|c|}
\hline $\begin{array}{c}\text { Tipos de } \\
\text { tecnologías de } \\
\text { impresión 3D }\end{array}$ & $\begin{array}{c}\text { Ejemplos de materiales para } \\
\text { procesos de aplicaciones médicas }\end{array}$ \\
\hline $\begin{array}{c}\text { Polimerización } \\
\text { Vat }\end{array}$ & $\begin{array}{c}\text { Una amplia variedad de polímeros } \\
\text { fotocurables }\end{array}$ \\
\hline $\begin{array}{c}\text { Fusión de } \\
\text { cama de } \\
\text { polvos }\end{array}$ & $\begin{array}{c}\text { Gran variedad de polímeros, } \\
\text { metales y materiales cerámicos. } \\
\text { Incluyendo PCL, HA, PLLA, } \\
\text { fosfato tricálcico. }\end{array}$ \\
\hline $\begin{array}{c}\text { Chorro de } \\
\text { material }\end{array}$ & $\begin{array}{c}\text { Variedad de polímeros y cerámicos, } \\
\text { incluyendo PCL, HA, vidrios } \\
\text { bioactivos, PLA, PEG. }\end{array}$ \\
\hline $\begin{array}{c}\text { Extrusión de } \\
\text { material }\end{array}$ & $\begin{array}{c}\text { Biopolímeros, polímero-cerámico y } \\
\text { compuestos de metalpolímero. }\end{array}$ \\
\hline $\begin{array}{c}\text { Deposición } \\
\text { directa de } \\
\text { energía }\end{array}$ & $\begin{array}{c}\text { HA/PLA, HA/PLC, vidrio } \\
\text { bioactivo. }\end{array}$ \\
\hline $\begin{array}{c}\text { Chorro de } \\
\text { aglutinante }\end{array}$ & $\begin{array}{c}\text { HA/PLA, HA/PCL, y vidrio } \\
\text { bioactivo. }\end{array}$ \\
\hline $\begin{array}{c}\text { Laminación de } \\
\text { hojas }\end{array}$ & $\begin{array}{c}\text { Variedad de metales, incluyendo } \\
\text { HA, zirconia, HA/MG63, células } \\
\text { humanas osteoprogenitoras y } \\
\text { células endoteliales. }\end{array}$ \\
\hline
\end{tabular}

La técnica empleada para la impresión 3D de determinada aplicación médica, dependerá de las características y necesidades de la misma, empleando aquella técnica que sea capaz de cumplir con las condiciones del modelo. Para esto la manufactura aditiva emplea datos mediante CT y MRI, contribuyendo a mejorar la precisión quirúrgica y otorgando mayor éxito. La tomografía computarizada parece ser la mejor opción para la extracción de datos durante la producción de modelos 3D debido a los diferentes valores en escala de unidades Hounsfield que corresponden a diferentes niveles de densidad de tejido [25, 31].

A continuación, se muestra el proceso empleado en el flujo de trabajo de una impresión 3D típica:

a. Selección de casos para impresión 3D (basado en la valoración clínica, costoefectividad y riesgos). b. Adquisición de la imagen (responsabilidad del radiólogo y médico especialista).

c. Segmentación (reconstrucción de la imagen, colaboración con técnico con conocimiento anatómico, especialista e ingeniero biomédico).

d. Ingeniería (desarrollo de modelos o guías, suavizado del modelo y apoyo en impresión).

e. Impresión 3D (ingeniero/ingeniero biomédico).

f. Preparación para uso (apoyo de ingeniero biomédico, con validación del especialista médico y si es necesaria la esterilización con el departamento central de servicio estéril).

Un ejemplo de una aplicación médica mostrada en el presente trabajo (Figura 4), es la fabricación de una prótesis de cadera personalizada. En este ejemplo se empleó una técnica de impresión basada en la fusión de cama de polvos, conocida como Sinterización Directa de Metales por Láser (DMLS) ya que este tipo de tecnología permite la fabricación de piezas metálicas con gran complejidad geométrica, las cuales podrían ser difíciles de manufacturar empleando técnicas convencionales. Los materiales que pueden emplearse son aleaciones metálicas, entre ellas las biocompatibles, como el acero inoxidable de grado médico, titanio, entre otros. Un tamaño de partícula entre 10 - 150 $\mu \mathrm{m}$ es preferible para obtener una alta resolución dimensional de las piezas [32]. 


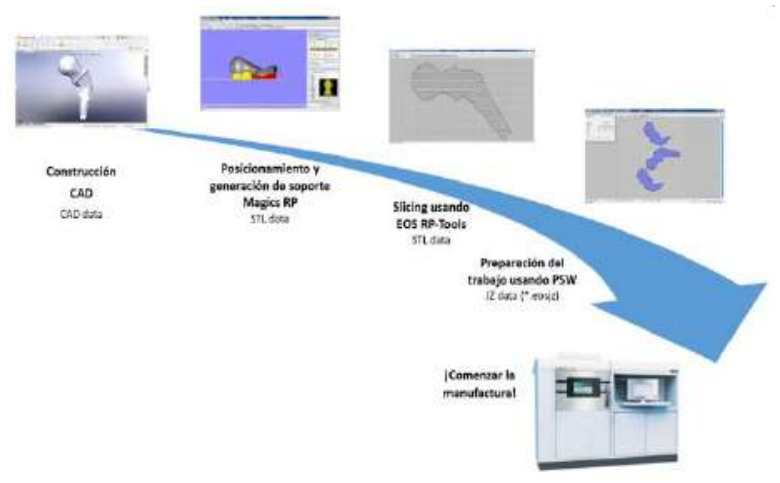

Figura 4. Proceso de manufactura empleado en la fabricación de un implante parcial de cadera personalizado mediante la técnica DMLS, impresora EOSINT M 280.

El proceso ejemplificado en la Figura 4 para la fabricación de una prótesis de cadera personalizada, se basa en el uso de la técnica DMLS empleando una impresora 3D de metales EOSINT M 280. Este procedimiento comprende 5 pasos generales:

1. La extracción del modelo en formato estereolitográfico STL.

2. La corrección del modelo y generación de soportes.

3. El proceso de slicing o verificación de la pieza capa por capa.

4. La preparación de las piezas para la impresión, su posición y parámetros de impresión.

5. La manufactura del modelo.

\section{CONCLUSIONES}

El éxito de un implante ortopédico está asociado con una correcta planeación preoperatoria. Para el proceso de personalización, las primeras etapas de identificación y estudio de la anatomía y necesidades del paciente determinan el tipo de prótesis y diseño a considerar. La personalización considera aspectos particulares de la prótesis para asemejarse tanto en propiedades como en dimensiones a la articulación que reemplaza.

La manufactura aditiva es una excelente herramienta para el diseño de prótesis personalizadas ya que a partir de imágenes médicas se pueden reproducir diseños de prótesis a medida del paciente. Existen aún retos tecnológicos y científicos en cada una de las etapas de la generación de prótesis personalizadas, desde la planeación hasta la fabricación.

\section{AGRADECIMIENTOS}

Agradecimientos al Consejo Nacional de Ciencia y Tecnología de México CONACYT a través del proyecto "Simposio Nacional de Prótesis Ortopédicas: El estatus Actual" y al programa de cátedras CONACYT a través del proyecto 848 .

\section{REFERENCIAS}

[1]. Kalaskar, Deepak M. (ed.). "3D printing in medicine”. Woodhead Publishing, 2017.

[2]. Brandt, Milan (ed.). "Laser additive manufacturing: materials, design, technologies, and applications". Woodhead Publishing, 2016.

[3]. Cronskär, Marie. "On customization of orthopedic implants-from design and additive manufacturing to implementation”. 2014. Tesis Doctoral. Kopieringen, Mid Sweden University.

[4]. Georgios Tsoulfas, Petros I. Bangeas, Jasjit Suri, "3D Printing: Applications in Medicine and Surgery”, Elsevier, 1a Ed., 2019. 
[5]. Shapií, A., Sulaiman, R., Hasan, M. K., Prabuwono, A. S., \& Kassim, A. Y. M. "Digital two dimensional (2D) implant design for pre-operative planning in Total hip Arthroplasty". International Journal on Electrical Engineering and Informatics, 4(1), 67, 2012.

[6]. Shichman, I., Factor, S., Shaked, O., Morgan, S., Amzallag, N., Gold, A., et al. "Effects of surgeon experience and patient characteristics on accuracy of digital preoperative planning in total hip arthroplasty". International Orthopaedics, 1-6, 2020.

[7]. Eggli, S., Pisan, M., \& Müller, M. E. "The value of preoperative planning for total hip arthroplasty". The Journal of bone and joint surgery. British volume, 80(3), 382-390, 1998.

[8]. Bono, J. V. "Digital templating in total hip arthroplasty”. JBJS, 86(suppl_2), 118122, 2004.

[9]. Bayne, C. O., Krosin, M., \& Barber, T. C. "Evaluation of the accuracy and use of $x$ ray markers in digital templating for total hip arthroplasty". The Journal of arthroplasty, 24(3), 407-413, 2009.

[10]. Della Valle, A. G., Comba, F., Taveras, N., \& Salvati, E. A. "The utility and precision of analogue and digital preoperative planning for total hip arthroplasty". International orthopaedics, 32(3), 289-294, 2008.

[11]. Holzer, L. A., Scholler, G., Wagner, S., Friesenbichler, J., Maurer-Ertl, W., \& Leithner, A. "The accuracy of digital templating in uncemented total hip arthroplasty". Archives of Orthopaedic and Trauma Surgery, 139(2), 263-268, 2017.

[12]. Kosashvili, Y., Shasha, N., Olschewski, E., Safir, O., White, L., Gross, A., \& Backstein, D. "Digital versus conventional templating techniques in preoperative planning for total hip arthroplasty". Canadian Journal of Surgery, 52(1), 6, 2009.

[13]. Konyves, A., \& Bannister, G. C. “The importance of leg length discrepancy after total hip arthroplasty". The Journal of bone and joint surgery. British volume, 87(2), 155157.

[14]. Sariali, E., Mauprivez, R., Khiami, F., Pascal-Mousselard, H., \& Catonné, Y. "Accuracy of the preoperative planning for cementless total hip arthroplasty. A randomised comparison between threedimensional computerised planning and conventional templating”. Orthopaedics \& Traumatology: Surgery \& Research, 98(2), 151-158, 2012.

[15]. Knafo, Y., Houfani, F., Zaharia, B., Egrise, F., Clerc-Urmès, I., \& Mainard, D. "Value of 3D Preoperative planning for primary total hip arthroplasty based on biplanar weightbearing radiographs". BioMed Research International, 2019.

[16]. Salem, H. S., Marchand, K. B., Ehiorobo, J. O., Tarazi, J. M., Matzko, C. N., Sodhi, N., et al. "Benefits of CT Scanning for the Management of Hip Arthritis and Arthroplasty". Surgical Technology International, 36, 2020.

[17]. Kobayashi, H., Cech, A., Kase, M., Pagenstart, G., Carrillon, Y., O'Loughlin, P. F., et al. "Pre-operative templating in THA. Part II: a CT-based strategy to correct architectural hip deformities". Archives of Orthopaedic and Trauma Surgery, 1-12, 2020 .

[18]. Yi, L. H., Li, R., Zhu, Z. Y., Bai, C. W., Tang, J. L., Zhao, F. C., et al. "Anatomical study based on 3D-CT image reconstruction of the hip rotation center and femoral offset in a Chinese population: preoperative implications in total hip arthroplasty". 
Surgical and Radiologic Anatomy, 41(1), 117-124. 2019.

[19]. Carter, L. W., Stovall, D. O., \& Young, T. R. "Determination of accuracy of preoperative templating of noncemented femoral prostheses". The Journal of arthroplasty, 10(4), 507-513, 2005.

[20]. Knight, J. L., \& Atwater, R. D. "Preoperative planning for total hip arthroplasty: quantitating its utility and precision". The Journal of arthroplasty, 7, 403-409, 1992.

[21]. Ahmad R, Sharma V, Sandhu H, Bishay M. "Leg length discrepancy in total hip arthroplasty with the use of cemented and uncemented femoral stems. A prospective radiological study”. Hip Int;19:264-7, 2009.

[22]. Sariali, E., \& Knaffo, Y. "Threedimensional analysis of the proximal anterior femoral flare and torsion. Anatomic bases for metaphyseally fixed short stems design". International Orthopaedics, 41(10), 2017.

[23]. Zeng, C., Xing, W., Wu, Z., Huang, H., \& Huang, W. "A combination of threedimensional printing and computer-assisted virtual surgical procedure for preoperative planning of acetabular fracture reduction". Injury, 47(10), 2223-2227, 2016.

[24]. Ganguli, A., Pagan-Diaz, G. J., Grant, L., Cvetkovic, C., Bramlet, M., Vozenilek, J., et al. "3D printing for preoperative planning and surgical training: a review". Biomedical microdevices, 20(3), 65, 2018.

[25]. Tsoulfas G., Petros I. Bangeas, Jasjit Suri, "3D Printing: Applications in Medicine and Surgery”. Elsevier, 1a Ed., 2019.
[26]. Lorna J.G., Michael F.A. "Cellular Solids. Structure and Properties" (Cambridge Solid State Science Series). Cambridge University Press. 2-26, 1999.

[27]. Schoen, A. H. "Infinite Periodic Minimal Surfaces Without Self-Intersection”. National Aeronautics and Space Administration (NASA) Tech, Washington, DC (Note no. D-5541), 1970.

[28]. Hussein, A., Hao, L., Yan, C. et al. "Advanced lattice support structures for metal additive manufacturing". Journal of Materials Processing Technology, 213. Pp 1019-1026, 2013.

[29]. Schileo, E., Dall'Ara, E., et al. "An accurate estimation of bone density improves the accuracy of subject-specific finite element models". Journal of Biomechanics, 41. Pp 2483-2491, 2008.

[30]. Kaneko, T.S. Bell, J.S., Pejcic, M.R., et al. "Mechanical properties, density and quantitative CT scan data of trabecular bone with and without metastases". J. Biomech, 37(4). Pp 523-530, 2004.

[31]. Deepak MK. "3D Printing in Medicine”. Elsevier. Chapters 1, 2. 1-42, 2017.

[32]. Duan B., Wang M., Zhou WY., Cheung WL., Li ZY., Lu WW. "Three-dimensional nanocomposite scaffolds fabricated via selective laser sintering for bone tissue engineering”. Acta Biomater. 6(12): 4495$505,2010$. 